\title{
Weighted Time-Variant Slide Fuzzy Time-Series Models for Short-Term Load Forecasting
}

\author{
Xiaojuan Liu ${ }^{1,2}$, Enjian Bai ${ }^{1}$, Jian'an Fang ${ }^{1}$ \\ ${ }^{1}$ College of Information Science \& Technology, Donghua University, Shanghai, China; ${ }^{2}$ Department of Mathematics and Physics, \\ Shanghai University of Electric Power, Shanghai, China. \\ Email: baiej@dhu.edu.cn
}

Received May $29^{\text {th }}, 2012$; revised June $26^{\text {th }}, 2012$; accepted July $3^{\text {rd }}, 2012$

\begin{abstract}
Short-term load forecast plays an important role in the day-to-day operation and scheduling of generating units. Season and temperature are the most important factors that affect the load change, but random factors such as big sport events or popular TV shows can change demand consumption in particular hours, which will lead to sudden load changes. A weighted time-variant slide fuzzy time-series model (WTVS) for short-term load forecasting is proposed to improve forecasting accuracy. The WTVS model is divided into three parts, including the data preprocessing, the trend training and the load forecasting. In the data preprocessing phase, the impact of random factors will be weakened by smoothing the historical data. In the trend training and load forecasting phase, the seasonal factor and the weighted historical data are introduced into the Time-variant Slide Fuzzy Time-series Models (TVS) for short-term load forecasting. The WTVS model is tested on the load of the National Electric Power Company in Jordan. Results show that the proposed WTVS model achieves a significant improvement in load forecasting accuracy as compared to TVS models.
\end{abstract}

Keywords: Load Forecasting; Fuzzy Time-Series; Weighted; Slide

\section{Introduction}

Load forecast has been a research topic for many decades and the accuracy of load forecast is crucial to electricity power industry due to its direct influence on generating planning. Short-term load forecast means the forecast time lead is in the range of hours to a few days ahead, which plays an important role in the day-to-day operation and scheduling of generating units. There are many factors that affect the load changes, such as calendar, weather, economical and random factors. For short-term load forecast, weather and random factors are the most important factors. Season and temperature are have the most influence to the load due to the fact that changes in temperature results in direct changes in energy consumption by heating and cooling appliances. Random factors such as big sport events or popular TV shows can change demand consumption in particular hours, which will lead to sudden load changes. A number of load forecasting models have been presented in the last decades. These models can be divided into traditional approaches [1] and the artificial intelligence methods [2]. The former include regression models, time series models et al, and the latter provided many new tools for the forecasting of shortterm load such as neural networks [3-5], fuzzy logic [6,7], support vector machines [8], expert systems [9], hybrid method $[10,11]$ et al. In recent years, many researchers have used fuzzy time series models to handle load forecasting problems [12-15]. Liu et al. proposed a Timevariant Slide Fuzzy Time-series Model (TVS) for shortterm load forecasting [13], the TVS model only uses historical data to predict the load changes. Taking into account the affect of season, temperature, and random factors, a Weighted Time-variant Slide Fuzzy Time-series Forecasting Model (WTVS) is presented. The WTVS model is divided into three parts, including the data preprocessing, the trend training and the load forecasting. In the data preprocessing stage, the impact of random factors will be weakened by smoothing the history data. In the trend training and load forecasting stage, the seasonal factor and the weight of history data are introduced into the TVS model. The WTVS model is tested on the load of the National Electric Power Company in Jordan. Results show that the WTVS model achieves a significant improvement in load forecasting accuracy as compared to TVS models.

\section{Time-Variant Fuzzy Time-Series}

A fuzzy set $A$ defined in the universe of discourse $U=\left\{u_{1}, u_{2}, \cdots, u_{n}\right\}$ can be represented as $A=f_{A}\left(u_{1}\right) / u_{1}+f_{A}\left(u_{2}\right) / u_{2}+\cdots+f_{A}\left(u_{n}\right) / u_{n}$, where $f_{A}$ is 
the membership function of the fuzzy set $A$, $f_{A}: U \rightarrow[0,1], f_{A}\left(u_{i}\right)$ denotes the degree of membership of $u_{i}$ be longing to the fuzzy set $A, f_{A}\left(u_{i}\right) \in[0,1]$, and $1 \leq i \leq n$.

Definition 1. Let $Y(t)(t=\cdots, 0,1,2, \cdots)$ be the universe of discourse and also a subset of $R$. It is assumed that $f_{i}(t)(i=1,2, \cdots)$ is defined on $Y(t)$ and $F(t)$ is the collection of $f_{i}(t)$, therefore, $F(t)$ is called a fuzzy time series on $Y(t)$.

Definition 2. It is assumed that $F(t)$ is a fuzzy time series and $F(t)=F(t-1) \times R(t, t-1)$, where $R(t, t-1)$ is a fuzzy relation and $\times$ is an operator which is caused by $F(t-1)$. The relationship between $F(t)$ and $F(t-1)$ can be denoted by $F(t-1) \rightarrow F(t)$ when $F(t)=F(t-1) \times R(t, t-1)$ is the first-order fuzzy timeseries model of $F(t)$.

Definition 3. Let $F(t)$ be a fuzzy time series. For any $t, F(t-1)=F(t)$ and $F(t)$ have only finite elements and therefore $F(t)$ is a time-invariant fuzzy time series; otherwise, it is a time-variant fuzzy time series.

Definition 4. If $F(t)$ is caused by

$F(t-1), F(t-2), \cdots, F(t-n)$, the fuzzy relationship is represented by $F(t-1), F(t-2), \cdots, F(t-n) \rightarrow F(t)$, it is the nth order fuzzy time-series model.

Definition 5. It is supposed that $F(t)$ is caused by $F(t-1), F(t-2), \cdots, F(t-m)(m>0)$, simultaneously and the relations are time variant. The $F(t)$ is a timevariant fuzzy time series and the relation can be expressed as $F(t)=F(t-1) \times R^{w}(t, t-1)$, where $w>1$ is a time parameter affecting the forecast $F(t)$, which is the analysis window of time-variant models.

\section{WTVS Model}

This study aims to improve short-term load forecasting using an adaptive algorithm to adjust the analysis window automatically in the training phase of weighted historical data and heuristic rules for forecasting in the testing phase. The WTVS model includes the following steps: 1) Preprocessing historical data, 2) defining and partitioning the universe of discourse, 3) defining fuzzy sets and fuzzifying time series, 4) establishing fuzzy relationships and 5) forecasting and defuzzifying forecasting results. These steps consist of three parts: preprocessing phase, training phase and testing phase. The preprocessing phase is used to eliminate the impact of random factors by smoothing the historical data. The training phase is used for data learning. Two values are computed in each round based on the selected analysis window sizes and the value with higher prediction accuracy is determined as the forecasting value. In this process, a sequence of the analysis windows is obtained. The selection of analysis window is determined by the following adaptive algorithm (Algorithm 1). The testing phase is used for forecasting accuracy test. Two values are computed by Algorithm 3 for every testing data based on the selected analysis window sizes of testing phase. Taking into account the affect of seasonal factor, a heuristic method is proposed to select the analysis window sizes of testing phase and determine the forecasting value based on the sequence of analysis window obtained in training phase. The structure of the WTVS model is presented in Figure 1.

In the following, details of each step is described.

Step 1. Preprocessing the historical load. Random factors may cause sudden load changes. We will smooth these sudden load changes by the following method when the absolute difference value of the load is higher than a threshold. The threshold is defined as

$$
\text { Threhold } \geq 3 \cdot \frac{\sum_{i=2}^{n}\left|F_{i}-F_{i-1}\right|}{n-1}
$$

Suppose that $\left|F_{i}-F_{i-1}\right| \geq$ Threshold, we will substitute $F_{t}$ by $F_{t-1}+$ Threshold.

Step 2. Fuzzified the revised historical load.

(1) Define the universe of discourse $U=\left[L_{\min }, L_{\max }\right]$ and separate it into $m$ intervals $u_{1}, u_{2}, \cdots, u_{m}$, $u_{i}=\left[L_{\min }+(i-1) l, L_{\min }+i l\right]$, where $l$ is the interval leng-

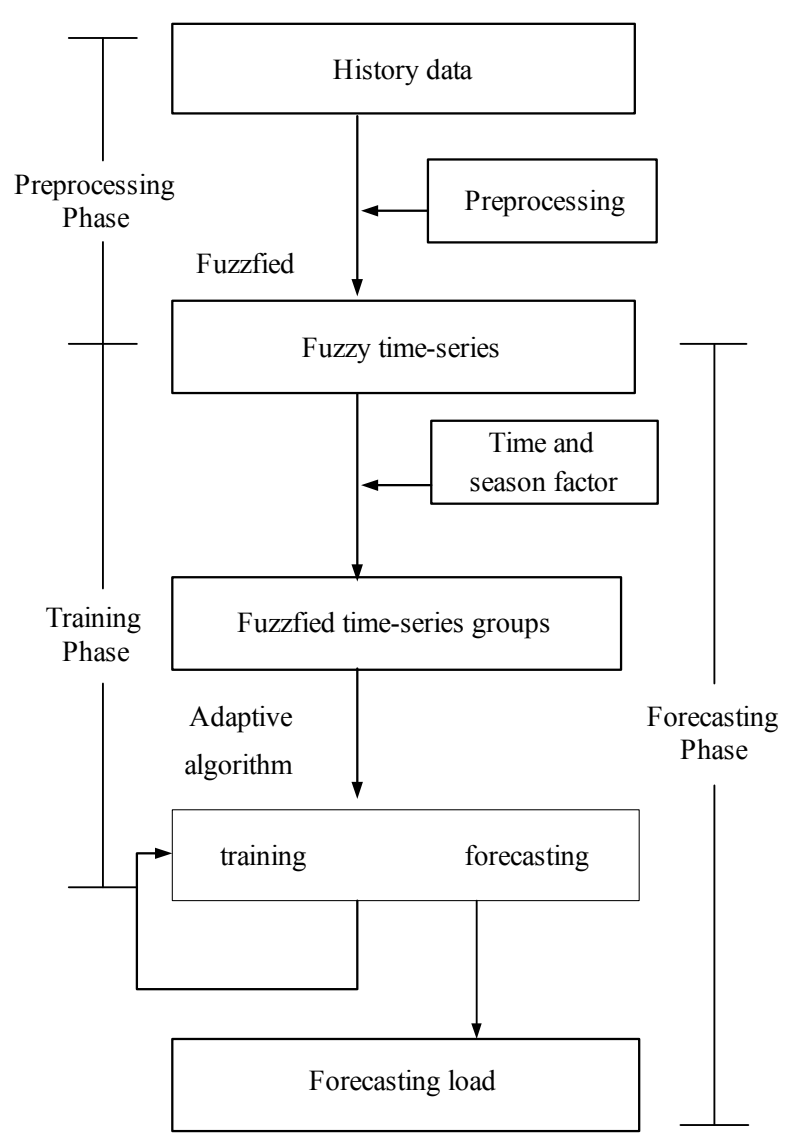

Figure 1. WTVS model. 
th, the midpoint of $u_{i}$ is $m_{i}$.

(2) Define the fuzzy sets $A_{i}$ and fuzzify the data.

$$
A_{i}=\frac{f_{A_{i}}\left(u_{1}\right)}{u_{1}}+\frac{f_{A_{i}}\left(u_{2}\right)}{u_{2}}+\cdots+\frac{f_{A_{i}}\left(u_{m}\right)}{u_{m}}(i=1,2, \cdots, m)
$$

Step 3. Establishing fuzzy relationships of time $t$ and $t+1$ and group the fuzzy time-series.

In the training phase, the fuzzy relationship is supposed to be $A_{i} \rightarrow A_{j}$. In the testing phase, the fuzzy relationship is supposed to be $A_{i} \rightarrow \#$.

Generally, the trend of load in summer and winter is shown in Tables 1 and 2, respectively. For example, in summer, we can conclude that from 1 to 6 o'clock, the load have the downward trend, while from 7 to 12 o'clock, the load have the upward trend. These trends can be used to revise the forecast in the forecasting phase.

Step 4. Forecasting and defuzzifying forecasting results.

In the training phase, each round calculates values For 1 and For 2 and compares the two values to actual value Act with the better one as the forecasting load. The analysis window is determined by Algorithm 1. The computations of For 1 and For 2 are carried out by Algorithm 2 . In the testing phase, the forecasting load is determined by Algorithm 3.

Algorithm 1. (slide analysis window)

(1) $i=1 ; S_{i}=1 ; S_{i+1}=2$, where $S_{i}$ and $S_{i+1}$ are the sizes of the initial window. Flag $n=1$.

(2) If the prediction accuracy computed by $S_{i+1}$ is higher than that of $S_{i}$, then slide the analysis window forward and the size of the analysis window plus 1 , and flag $n=n+1$. Otherwise, slide the analysis window backward and the size of the analysis window minus 1 , and flag $n=n-1$.

(3) Repeat step (2) until the end of the training data.

Algorithm 2. (training phase) Suppose that the fuzzy relationship of time $k$ and $k+1$ is $A_{i} \rightarrow A_{j}$, and the analysis window size is $n$. Let $M\left[A_{j}\right]$ be the middle value of interval $u_{j}$.

(1) Select two initial window sizes $S_{1}=1$ and $S_{2}=2$. Let $n=1$.

(2) If $n=1$, For $1=M\left[A_{j}\right]$. If $n \geq 2$,

Table 1. Load trend in summer.

\begin{tabular}{ccccc}
\hline Time & $1-6$ & $7-12$ & $13-20$ & $21-24$ \\
Trend & $\downarrow$ & $\uparrow$ & $\downarrow$ & $\downarrow$ \\
\hline
\end{tabular}

Table 2. Load trend in winter.

\begin{tabular}{cccccc}
\hline Time & $1-6$ & $7-12$ & $13-16$ & $17-18$ & $19-24$ \\
Trend & $\downarrow$ & $\uparrow$ & $\downarrow$ & $\uparrow$ & $\downarrow$ \\
\hline
\end{tabular}

For 2

$$
=\frac{\sum_{\lambda \in \Lambda}\left[F_{t}+\lambda \sum_{i=0}^{n-2}\left(\frac{2}{n}-i \frac{2}{n(n-1)}\right)\left|F_{t-i}-F_{t-i-1}\right|\right]+M\left[A_{j}\right]}{s+1}
$$

where $\lambda \in\left\{ \pm \frac{1}{8}, \pm \frac{1}{6}, \pm \frac{1}{4}, \pm \frac{1}{2}, \pm 1, \pm 2, \pm 3, \pm 4\right\} \triangleq \Lambda$

and $F(t)$ is the actual load at time $t$.

$$
\text { If } D \triangleq F_{t}+\lambda \sum_{i=0}^{n-2}\left(\frac{2}{n}-i \frac{2}{n(n-1)}\right)\left|F_{t-i}-F_{t-i-1}\right| \in u_{j}
$$

then $\lambda \in \Lambda$, and $s=s+1$. Else $\lambda \notin \Lambda$, and $s=s$.

(3) Compared with the actual load, if the training accuracy of the For 2 is higher than For 1 , then $F_{\text {predict }}=$ For 2 . Else $F_{\text {predict }}=$ For 1 .

(4) Slide the analysis window until the end of the whole training data.

Algorithm 3. (forecasting phase ) Suppose that the fuzzy relationship of time $k$ and $k+1$ is $A_{i} \rightarrow \#$, and the analysis window size is $n$. let $M\left[A_{j}\right]$ be the middle value of interval $u_{j}$.

(1) Select two initial window sizes $S_{1}=1$ and $S_{2}=2$. Let $n=1$.

(2) If $n=1$, For $3=M\left[A_{i}\right]$. If $n \geq 2$,

$$
\text { For } 4=\frac{\sum_{\lambda \in \Lambda}\left[F_{t}+\lambda \sum_{i=0}^{n-2}\left(\frac{2}{n}-i \frac{2}{n(n-1)}\right)\left|F_{t-i}-F_{t-i-1}\right|\right]}{s},
$$

where $\lambda \in\left\{ \pm \frac{1}{8}, \pm \frac{1}{6}, \pm \frac{1}{4}, \pm \frac{1}{2}, \pm 1, \pm 2, \pm 3, \pm 4\right\}^{\Delta}=\Lambda$

and $F(t)$ is the actual load at time $t$.

$$
\text { If } D \triangleq F_{t}+\lambda \sum_{i=0}^{n-2}\left(\frac{2}{n}-i \frac{2}{n(n-1)}\right)\left|F_{t-i}-F_{t-i-1}\right| \in u_{j} \text {, }
$$

Then $\lambda \in \Lambda$, and $s=s+1$. Else $\lambda \notin \Lambda$, and $s=s$.

(3) Consider the trend of load change and the sequence of flags $n$ which obtained in training phase, there are the following heuristic rules:

1) If $n_{t}>n_{t-1}$, and the actual load at time $t$ is bigger than that of time $t-1$, then the load at time $t+1$ has the trend of increasing. At the same time pay attention to the trend in Tables $\mathbf{1}$ and 2, the forecast value is max \{For 3 For 4$\}$.

2) If $n_{t}<n_{t-1}$, and the actual load at time $t$ is smaller than that of time $t-1$, then the load at time $t+1$ has the trend of decreasing. At the same time pay attention to the trend in Tables 1 and 2, the forecast value is $\min$ \{For 3 For 4 \}.

3) Else, the forecast value is the arithmetic average of For 3 and For 4. 
(4) Slide the window until the end of the whole forecasting data.

\section{Experiments and Analysis}

The load of the National Electric Power Company in Jordan [12] is chosen for model validation. An empirical analysis is conducted to validate the performance of WTVS model by comparing the forecasted load with that of TVS model [13]. Considering the time and season factors, we choose the data from 1 to 24 in each day as our research data. The data are divided into two parts: the training data (from 1 to 20 ) and the forecasting data (from 21 to 24). Table 3 lists the load in 23/5 and 29/6 and the corresponding revised load by preprocessing. For the purpose of the comparisons of the predictive accuracy, we use the mean absolute percentage error (MAPE) as the index of forecasting accuracy. MAPE can be defined as

$$
\text { MAPE }=\frac{1}{n} \sum_{k=1}^{n}\left|\frac{t_{k}-m_{k}}{t_{k}}\right| \times 100 \%
$$

where $t_{k}, m_{k}$ represent the actual and forecasting values of the $k$ th data, respectively. And $n$ is the number of data. Table 4 compares load forecasting results among WTVS and VTS model in the forecasting phase with the same number of intervals. The MAPE results show WTVS model outperforms TVS model.

Table 5 shows the different forecasting accuracy in

Table 3. Load preprocessing.

\begin{tabular}{|c|c|c|c|c|c|c|}
\hline & 23/5 Actual load & $F_{t+1}-F_{t}$ & 23/5 Revised load & 29/6 Actual load & $F_{t+1}-F_{t}$ & 29/6 Revised load \\
\hline 1 & 1176 & & 1176 & 1285 & & 1285 \\
\hline 2 & 1129 & -47 & 1129 & 1210 & -75 & 1210 \\
\hline 3 & 1095 & -34 & 1095 & 1170 & -40 & 1170 \\
\hline 4 & 1098 & 3 & 1098 & 1130 & -40 & 1130 \\
\hline 5 & 1093 & -5 & 1093 & 1145 & 15 & 1145 \\
\hline 6 & 1080 & -13 & 1080 & 1080 & -65 & 1080 \\
\hline 7 & 1195 & 115 & 1195 & 1140 & 60 & 1140 \\
\hline 8 & 1327 & 132 & 1327 & 1360 & 220 & 1350 \\
\hline 9 & 1509 & 182 & 1509 & 1485 & 125 & 1485 \\
\hline 10 & 1567 & 58 & 1567 & 1548 & 63 & 1548 \\
\hline 11 & 1614 & 47 & 1614 & 1612 & 64 & 1612 \\
\hline 12 & 1640 & 26 & 1640 & 1640 & 28 & 1640 \\
\hline 13 & 1610 & -30 & 1610 & 1631 & -9 & 1631 \\
\hline 14 & 1600 & -10 & 1600 & 1600 & -31 & 1600 \\
\hline 15 & 1591 & -9 & 1591 & 1605 & 5 & 1605 \\
\hline 16 & 1547 & -44 & 1547 & 1565 & -40 & 1565 \\
\hline 17 & 1528 & -19 & 1528 & 1486 & -79 & 1486 \\
\hline 18 & 1482 & -46 & 1482 & 1420 & -66 & 1420 \\
\hline 19 & 1418 & -64 & 1418 & 1380 & -80 & 1380 \\
\hline 20 & 1700 & 282 & 1628 & 1535 & 155 & 1535 \\
\hline 21 & 1633 & -67 & 1633 & 1615 & 80 & 1615 \\
\hline 22 & 1515 & -188 & 1515 & 1520 & -95 & 1520 \\
\hline 23 & 1417 & -98 & 1417 & 1475 & -45 & 1475 \\
\hline 24 & 1293 & -124 & 1293 & 1370 & -105 & 1370 \\
\hline Average & & 68.4 & & & 67.2 & \\
\hline Threshold & & 210 & & & 210 & \\
\hline
\end{tabular}


Table 4. Comparison between WTVS and TVS model in forecasting phase.

\begin{tabular}{cccccccc}
\hline $23 / 5$ & $\begin{array}{c}\text { Actual } \\
\text { load }\end{array}$ & WTVS & TVS $^{[3]}$ & $29 / 6$ & $\begin{array}{c}\text { Actual } \\
\text { load }\end{array}$ & WTVS & TVS $^{[3]}$ \\
\hline 21 & 1633 & 1620 & 1723 & 21 & 1615 & 1547 & 1519 \\
22 & 1515 & 1625 & 1607 & 22 & 1520 & 1620 & 1627 \\
23 & 1417 & 1497 & 1538 & 23 & 1475 & 1529 & 1525 \\
24 & 1293 & 1419 & 1433 & 24 & 1370 & 1460 & 1475 \\
MAPE & & 5.8 & 7.74 & & & 5.2 & 6.0 \\
\hline
\end{tabular}

Table 5. Comparison under different numbers of intervals in forecasting phase.

\begin{tabular}{cccccc}
\hline \multirow{2}{*}{ Time } & \multicolumn{5}{c}{ Number of intervals } \\
\cline { 2 - 6 } & 4 & 5 & 8 & 10 & 16 \\
\hline $23 / 5$ & 6.94 & 7.23 & 7.74 & 8.27 & 8.18 \\
$29 / 6$ & 4.8 & 7.28 & 4.6 & 5.87 & 5.43 \\
\hline
\end{tabular}

the forecasting phase under different numbers of intervals. It is shown that the forecast accuracy is influenced by the length of intervals.

\section{Conclusions}

In this paper a weighted time-variant slide fuzzy timeseries model for short-term load forecasting is proposed. The proposed model is tested for forecasting efficacy on the load of the National Electric Power Company in Jordan. Some of the heuristic knowledge generated by the WTVS model in the training phase is used to forecast unknown future values. The experimental results show that the WTVS model is more accurate than TVS model. The advantages of the WTVS model are as follows.

1) External factors were considered in WTVS model. In the data preprocessing phase, the impact of random factors is weakened by smoothing the historical data. In the trend training and load forecasting phase, the seasonal factor was introduced into TVS model.

2) The most recent data from the prediction load has the greater impact. The weighted historical data are considered in WTVS model.

\section{Acknowledgements}

The authors would like to thank the anonymous reviewers and the work was supported by Shanghai Municipal Natural Science Fund under grant 10ZR1401400 and The Fundamental Research Funds for the Central Universities under grant 11D10417 and 11D10402.

\section{REFERENCES}

[1] M. T. Hagan and S. M. Behr, "The Time Series Approach to Short Term Load Forecasting," IEEE Transactions on Power Systems, Vol. 2, No. 3, 1987, pp. 785-791. doi:10.1109/TPWRS.1987.4335210

[2] H. Hahn, S. M. Nieberg and S. Pickl, "Electric Load Forecasting Methods: Tools for Decision Making," European Journal of Operational Research, Vol. 199, No. 3, 2009, pp. 902-907. doi:10.1016/j.ejor.2009.01.062

[3] J. W. Taylor and R. Buizza, "Neural Networks Load Forecasting with Whether Ensemble Predictions," IEEE Transactions on Power Systems, Vol. 17, No. 3, 2002, pp. 626-630. doi:10.1109/TPWRS.2002.800906

[4] Y. Chen, P. B. Luh and C. Guan, "Short-term Load Forecasting: Similar Day-Based Wavelet Neural Networks," IEEE Transactions on Power Systems, Vol. 25, No. 1, 2010, pp. 322-330. doi:10.1109/TPWRS.2009.2030426

[5] A. S. Pandey, D. Singh and S. K. Sinha, "Intelligent Hybrid Wavelet Models for Short-Term Load Forecasting," IEEE Transactions on Power Systems, Vol. 25, No. 3, 2010, pp. 1266-1273. doi:10.1109/TPWRS.2010.2042471

[6] A. Khotanzad, Z. Enwang, and H. Elragal, "A Neurofuzzy Approach to Short-term Load Forecasting in a Price-sensitive Environment," IEEE Transactions on Power Systems, Vol. 17, No. 4, 2002, pp. 1273-1282. doi:10.1109/TPWRS.2002.804999

[7] V. H. Hinojosa and A. Hoese, "Short-term Load Forecasting Using Fuzzy Inductive Reasoning and Evolutionary Algorithms," IEEE Transactions on Power Systems, Vol. 25, No. 1, 2010, pp. 565-574. doi:10.1109/TPWRS.2009.2036821

[8] C. Bo-juen, C. Ming-Wei and L. Chih-jen, "Load Forecasting Using Support Vector Machines: a Study on EUNITE Competition 2001," IEEE Transactions on Power Systems, Vol. 19, No. 4, 2004, pp. 1821-1830. doi:10.1109/TPWRS.2004.835679

[9] D. Fay and J. V. Ringwood, "On the Influence of Weather Forecast Errors in Short-Term Load Forecasting Models," IEEE Transactions on Power Systems, Vol. 25, No.3, 2010, pp. 1571-1758. doi:10.1109/TPWRS.2009.2038704

[10] K. B. Song, S. K. Ha and J. W. Park, "Hybrid Load Forecasting Method with Analysis of Temperature Sensitivities," IEEE Transactions on Power Systems, Vol. 21, No. 2, 2006, pp. 869-876. doi:10.1109/TPWRS.2006.873099

[11] S. Fan and L. U. Chen, "Short-Term Load Forecasting Based on an Adaptive Hybrid Method," IEEE Transactions on Power Systems, Vol. 21, No. 1, 2006, pp. 392401. doi:10.1109/TPWRS.2005.860944

[12] R. Mamlook, O. Badran and E. Abclulhadi, "A Fuzzy Inference Model for Short Term Load Forecasting," Energy Policy, Vol. 37, No. 4, 2009, pp. 1239-1248. doi:10.1016/j.enpol.2008.10.051

[13] X. J. Liu, E. J. Bai and J. Fang, "Time-Variant Slide Fuzzy Time-series Method for Short-Term Load Forecasting," Proceeding of 2010 IEEE International Conference on Intelligent Computing and Intelligent Systems, Xiamen, 29-31 October 2010, pp. 65-68.

[14] H. T. Liu, N. C. Wei and C. G. Yang, "Improved TimeVariant Fuzzy Time Series Forecast," Fuzzy Optimization and Decision Making, Vol. 8, No. 1, 2009, pp. 45-65. 
doi:10.1007/s10700-009-9051-8

[15] C. A. Maia and M. Goncalves, "Application of Switched Adaptive System to Load Forecasting," Electric Power
Systems Research, Vol. 78, No. 4, 2008, pp. 721-727. doi:10.1016/j.epsr.2007.05.014 